\title{
DETERMINING THE VALIDITY OF FURUE'S METHOD OF CRANIOFACIAL SUPERIMPOSITION FOR IDENTIFICATION
}

B. Scully, P. Nambiar. Determining the validity of Furue's method of craniofacial superimposition for identification. Annal Dent Univ Malaya 2002; 9: 1722.

\begin{abstract}
Craniofacial superimposition methods are employed for the identification of unknown skulls or living persons. There are many such methods and of particular interest is that technique developed by Furue which is inexpensive to set-up. A study was undertaken to ascertain the validity of this technique and to correlate our findings with other researchers.
\end{abstract}

Key words: Craniofacial superimposition, identification, Furue's method.

\section{INTRODUCTION}

The human face is a reflection of who we are, how we feel, and where we come from, what we do and much more. It can be a record of our lives and even in death it can be employed in identifying who we are. There are many ways of identifying missing unknown persons. This includes from antemortem dental records, personal effects, photographic and video images and DNA studies. New methods are constantly being developed which along with refinements to existing techniques are able to satisfy the medico-legal and humanitarian requirements for identification.

Superimposition is defined as, to set or place on or over something else. Craniofacial superimposition works as an identification technique for the dead as well as for the living. This is achieved either by laying an image of the skull or face of a person over the image of a known person. Therefore, craniofacial photographic superimposition is a method of identification achieved by the comparison of an image of a skull overlaid upon an antemortem photograph of a missing person, thought likely to be the same subject. This form of identification may also be extended where the photographic image of a missing person could be superimposed onto a reconstructed face from an unknown excavated skull. Similarities in anatomical features and soft tissue averages are observed to achieve a possible identification. Furthermore, superimposition in its earliest form was done by hand drawn tracings, but this technique progressed to the utilisation of still photography, video technology and lately computers.

This project and research was undertaken to setup a relatively inexpensive craniofacial photographic
Original Article

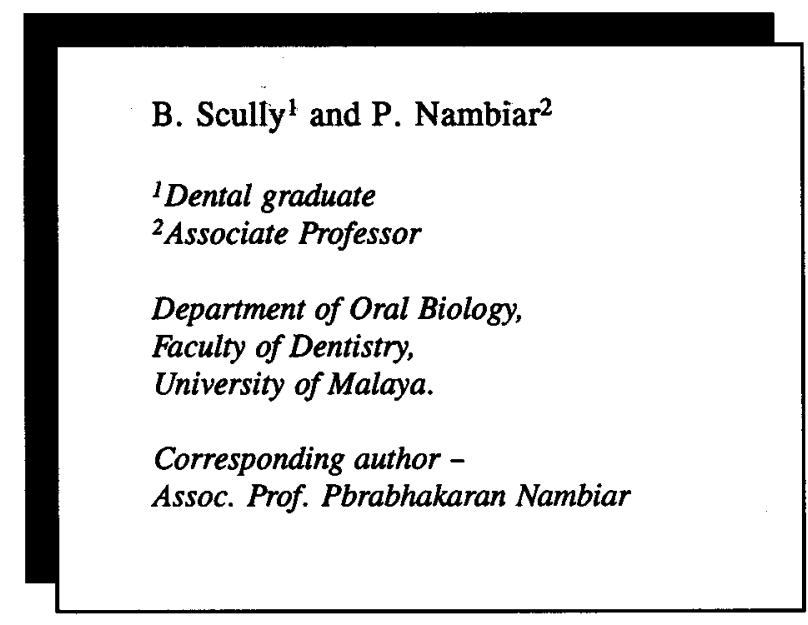

superimposition facility, based on Furue's method at the Faculty of Dentistry, University of Malaya. Subsequently we wanted to test the reliability of this technique for forensic applications. By analysis of past studies, their results, and correlating our findings, it was hoped that this craniofacial superimposition method of identification can be employed in the future.

\section{HISTORICAL BACKGROUND}

The method of craniofacial identification from portraits, sculptured bust and from death masks has been documented as early as the late eighteen century. One of the earliest pioneers was Hermann Welker, a German scientist who analysed the measurements of a skull said to be that of the famous poet Dante with his death mask kept in Florence, Italy $(1,2,3)$. Lander in 1918 was the first person to use the antemortem photographic comparison method with a skull. However, superimposition of the skull and the said photograph was not performed. In the early years of the 20th century, Pearson and Morant in England compared the skulls supposedly of famous people, notably Jeremy Bentham, Sir Thomas Browne, Robert the Bruce, George Buchanan, Lord Darnley and Oliver Cromwell with their portraits, busts, or death masks $(1,2,3)$.

The first documented use of the craniofacial superimposition technique in a criminal case was by Glaister and Brash in 1937 (4) in a case whereby they identified two mutilated and dismembered bodies found in a Scottish riverbed. It was used as corroborative evidence and was not used as the primary means of identification. In their work, life sized antemortem photographs of the victims and life sized photographs of the skulls were used. Cocks (5) used superimposition to identify the fragmented skull of a murder victim. A photograph of the reconstructed skull 
was compared with the passport photograph of the suspected victim. A triangulation system was used to demonstrate the degree of similarity seen between the skull and the photograph.

Thomas, Nortjé and van Ieperen (6) superimposed one projected image onto a photographic print to achieve a comparison. They enlarged a passport photograph of the suspected victim to a live sized image and fixed this in the vertical plane. A transparency of the skull in the similar orientation was then projected onto the image. The projector was placed at a distance that permitted projection of a lifesized image of the skull. The report does not indicate if any consideration was given to problems associated with perspective in either recording of the image of the skull or the projection of it onto the antemortem photograph.

The most important contribution of craniofacial superimposition using still photography was devised by Furue and used by the United States Armed Forces Central Identification Laboratory since 1971. Our current work is based on this method. Apart from craniofacial superimposition, Klonaris and Furue (7) applied the superimposition method to the comparison of a maxillary fragment in a dental radiograph. The victim was a pilot serving in the Vietnam War.

In 1983, Brown (8) introduced a significant modification to the photographic craniofacial superimposition. They substituted the use of still cameras with a video camera system, thereby eliminating the developing and printing of repeated photographic exposures to obtain the correct orientation and enlargement. Two video cameras were employed, one focused on the antemortem photograph and the other on the skull. Each camera transmitted its image to a separate monitor and both image signals were then fed through a special effects generator and superimposed together to a third monitor. The special effects generator was used to create a variety of image combinations. The video recordings of the superimposition process could be made for further study and presentation in courts.

\section{MATERIALS AND METHOD}

\section{(A) SETTING-UP THE CRANIOFACIAL SUPERIMPOSITION FACILITY}

The first part of the project was to set-up the craniofacial superimposition facility according to Furue's method using mirrors, panning motor, light, lighting controls, stands and grids (9). The arrangement and set-up of the equipment used is presented in Fig.1.

\section{Camera:}

The cameras used were an automatic Canon camera, Canon EOS 808 SLR camera and a Nikon SLR F50 camera, mounted on a tripod. Of the two camera lenses used, one was a Canon zoom lens with the focal range $35-130 \mathrm{~mm}$ and the other was a Tamron brand zoom lens, with a focal length of 28 $200 \mathrm{~mm}$.

\section{Skull mounting:}

The supplied parts were attached together from the following components: -

i) King brand electronic motorised pan head MP101 with remote control.

ii) Kaiser brand ball and socket head joint.

iii) Hama brand binoculars clamp.

The completed clamp was then inserted through the foramen magnum of the skull. The clamp was then tightened with tissue papers to soften the clamping pressure and enhance the grip of the clamp onto the skull. This apparatus allowed the mechanically controlled movement of the skull in 3 axes. A remote control unit was used for fine exacting movements.

\section{Mirrors:}

One two-way mirror (half-silvered mirror) and one single way mirror (full optical mirror) are the original components of the superimposition system. The mirrors were framed and then attached to a level piece

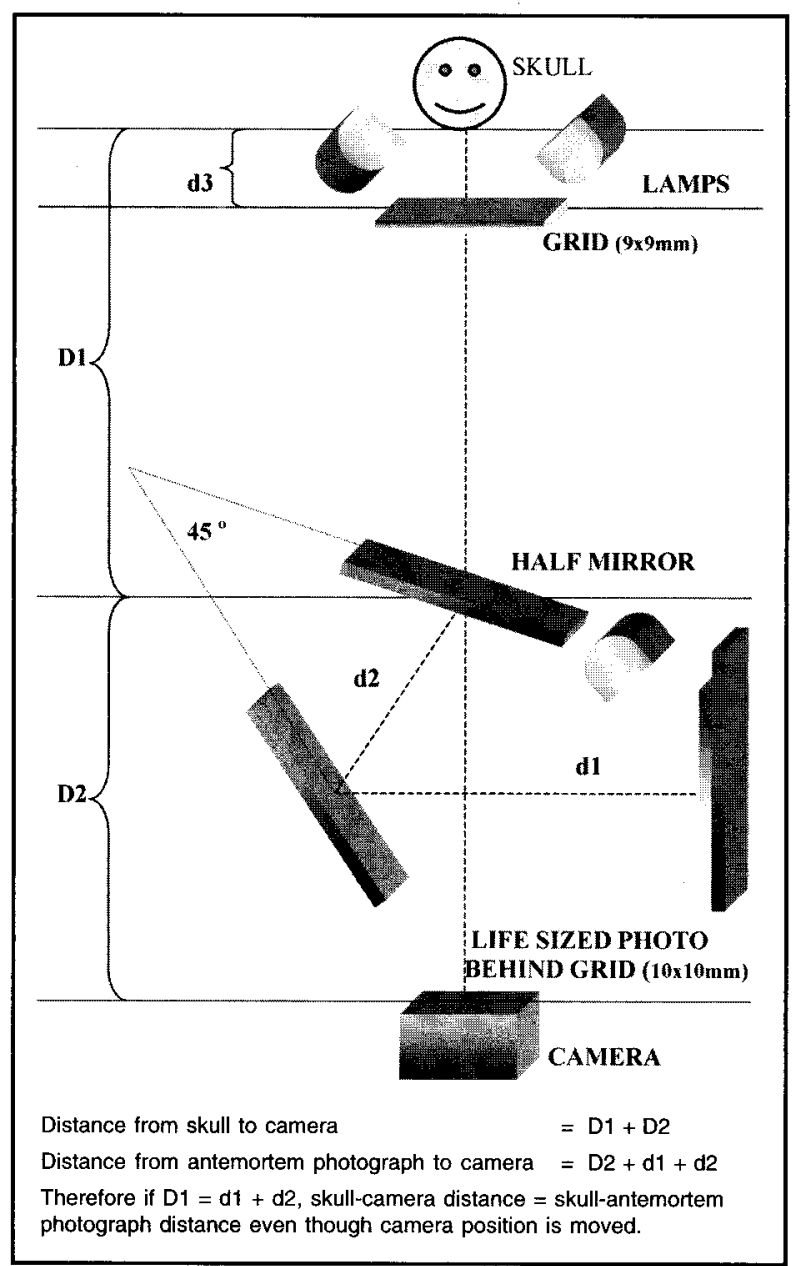

Figure 1: Layout of the superimposition equipment. 
of wood with a hinge, which was able to be manipulated to 90 degrees perpendicular to the framed mirrors. When the wood support was opened, this functioned as a stand, which positioned the mirrors vertically.

\section{Lighting:}

Two separate focusing light sources with separate controls were designed to give ambient lighting. Ambient lighting means the ability to amplify or reduce the intensity of the lighting of both the skull as well as the antemortem photograph. The intensity of light to be used was dependent on whether the skull or the antemortem photograph needed to be highlighted and made more visible or if they were made to be merged into one superimposed image.

\section{Grids:}

Two grids were prepared using Kodak photocopy transparency sheets and framed with glass. One of the grid had squares measuring $9 \mathrm{mmX} 9 \mathrm{~mm}$ and the other was $10 \mathrm{mmX} 10 \mathrm{~mm}$.

\section{Stands:}

A stand was made for mounting the antemortem photograph vertically with $10 \mathrm{~mm} \times 10 \mathrm{~mm}$ grid directly in front of the photograph. Another stand was made for the vertical placement of the $9 \mathrm{~mm} \mathrm{X} 9 \mathrm{~mm}$ grid close to the position of the mounted skull. This position is at $90 \%$ of the distance of the skull from the camera.

The film used for this project was standardised as Kodak Gold 200-colour film with 36 exposures per roll. The ASA 200 film was chosen for use since its characteristics are intermediate between the "slow" films (ASA 100) and "fast" films (ASA 400). This allows for both the qualities, such as wide exposure latitude (allowing for good pictures even with exposure error) and sharp images as the emulsion grains are sufficiently fine.

The film once used was sent for development and printing at a Kodak photographic shop using only Kodak equipment and paper for conformity. The prints were made in $3 \mathrm{R}$ size and scanned into a computer using the Genius Colourpage Vivid III scanner. The scanned photographs were then enlarged to their life size employing the ruler in the photograph.

\section{Computer and software programmes:}

A pentium computer and a 233 MMX computer was used to manipulate the scanned images from the photographs taken of the skulls. These images were scanned with a Genius Colorpage Vivid scanner, with 48-bit superior colour enhancement technology, optical resolution of $600 \mathrm{X} 1200 \mathrm{dpi}$ (dots per inch) resolution, with possible interpolation of up to $19200 \mathrm{dpi}$. The program used to scan the photographs was Easyphoto 2.7, and Adobe Photodeluxe. The scanned file was in the rich Photodeluxe format and edited with the Adobe Photoshop 5.5 program to enlarge and clarify the image to life size. The printer used was the Epson Stylus Color 460, with high resolution up to $720 \times 720$ dpi using micro piezo technology for fine details and picture clarity. Super high-grade color inkjet paper from Mitsubishi Chemical Labs of Japan was used for achieving better contrast.

\section{(B) THE VERIFICATION OF THE VALIDITY OF FURUE'S METHOD OF CARNIOFACIAL SUPERIMPOSITION}

The second part of this project involved the testing of the set-up facility to verify the validity of Furue's method, to check the effectiveness of the method and also to identify any weakness in this system.

A skull was photographed under conditions similar to those commonly found in ordinary photographs, for example in an indoor setting (for example natural light or a typical household light source). Pictures of the skull were taken with the automatic camera. These pictures were taken at increasing distances of $1 \mathrm{~m}, 2 \mathrm{~m}$, $3 \mathrm{~m}, 4 \mathrm{~m}, 5 \mathrm{~m}, 6 \mathrm{~m}$, and $7 \mathrm{~m}$. Pictures of the skull were also taken with the Canon and Tamron zoom lenses which were adjustable to provide for a large and clear image of the skull within the viewfinder. Therefore an adequately large image of the skull was reproduced in the resulting photograph. These pictures were also taken at increasing skull-camera distances of $1 \mathrm{~m}, 2 \mathrm{~m}$, $3 \mathrm{~m}, 4 \mathrm{~m}, 5 \mathrm{~m}, 6 \mathrm{~m}$, and $7 \mathrm{~m}$.

To test the reliability and accuracy of the various components and also the set-up, two identical prints were made. These two identical pictures were then mounted on the two stands (as for the skull and the antemortem photograph) with the grids to align the placement. These two images were superimposed onto one another and observed for any discrepancies. There were no noticeable differences found when comparing the identical sets of images. Therefore the set-up was verified and taken to be correct and able to be used for further comparisons.

The following aspects were further studied:

1) The effect of the camera lenses used and the level of distortion produced.

2) The effect of focal length on the photographic image produced.

3) The effects of camera - subject distances on the photographic image.

Subsequently the comparisons between the skull and the printed life-size image were done according to a set guideline. Since it was the comparison between a three-dimensional real life object (i.e. skull) and its two dimensional recreated image, it could not be expected for all features to match perfectly in the superimposition. This was because the photograph was able to reproduce the skull in a two dimensional view only. Therefore, only landmarks and features on the skull found in the same plane of view would match 
the real life skull in an exact orientation. The most common features used to subjectively ascertain the correspondence of features of the skull were as follows; the shape of the skull in general, the shape of the orbit, the nasal aperture, the external auditory meatus, the zygomatic arch and the teeth when they are visible.

The life-sized prints were also manipulated and spliced using the Adobe Photoshop 5.5. program into halves and placed side by side with the other halves taken at different settings. This enabled to determine any deviation from matching of anatomical landmarks between both halves taken at different settings.

\section{RESULTS}

1) Comparison was made using the pictures of the skull taken at the same distance, but taken using two different lenses. It was found that there were no noticeable differences in this comparison. This proves that when the subject was parallel with the focal plane of the camera, the use of different lenses has no influence on the superimposition of the photographic images.

2) Comparison was made of skull photographs that were taken from the same distance using the zoom lens but using different focal lengths. The images were then enlarged to the same life-size and compared. Although the size of the images in the original photographs were different depending on the focal lengths of the lens used, when enlarged to the equivalent size, they were found to be identical, demonstrating that the lens focal length has no effect on the photographic image characteristics.

3) The skull was then photographed at different camera-subject distances but using the same focal length lens (Canon automatic camera with fixed lens). The photographs were then enlarged to the same life-size and then compared by placing besides each other. It was found that the image differed with each distance change, therefore bringing us to the conclusion that each photograph was characterised by its own perspective, which was dependant on the distance between the camera and the subject.

In addition, photographs were then taken from different camera positions and the images compared. It was found that by changing the camera positions alters the relative orientation of the skull to the lens and introduces a parallax error, which is unacceptable for any superimposition procedures.

When comparing the features of the skull to photograph, the general shape of the skull was found not suitable for comparison. This was because the lower jaw becomes anteriorly placed and the vertex and mastoids posteriorly placed. These parts and likewise certain other features of the skull were not in the same viewing plane as this was still a three dimensional object. Therefore this could lead to a misjudgement on the part of the operator during subjective appraisal when trying to establish correspondence of features on the photograph to the skull during the superimposition procedures.

\section{DISCUSSION}

This study allowed us to compare the reliability of digitally scanned and enlarged images to the conventional method of direct photographic prints developed to life size from negatives. The results showed that despite using digital images the findings were similar to the conventional direct photographic prints.

\section{Perspective}

Perspective is defined as the two dimensional representation of size, shape and position of objects as they exist in their three dimensional state (10). Perspective distortion is not a product of the lens but by the distance from the subject to be photographed. Lenses with short and long focal lengths do not produce the same image on film when they are used at the same distance from the subject. In practice, the longer the focal length, the more compressed the subjects at varying distances will appear. In other words, objects far from the camera will appear greater in size in relationship to the foreground size.

Differences in photographs taken from distances greater than $5 \mathrm{~m}$ were found to be negligible since perspective from that distance was compressed and thus approximately similar. Consequently when applying superimposition to personal identification, it is necessary to know the camera subject distance from which the antemortem photograph was taken. In practice, if the antemortem photograph was taken at a distance more than $5 \mathrm{~m}$, then it is sufficient to photograph the skull from $5 \mathrm{~m}$. If the photograph was taken at a distance less than $5 \mathrm{~m}$, the camera skull distance must be adjusted accordingly for superimposition procedures.

\section{Distance}

As the camera - subject distance is a factor significantly affecting image characteristics, the superimposition apparatus must include a provision for alteration of camera to subject (skull) distance in order to duplicate the distance at which the antemortem photograph was taken.

A photograph forms a permanent non-changing recording of the two dimensional state. From the same point, at the same distance, all lenses will produce a photograph with the same perspective. The focal length of the lenses will only contribute to the size of the image seen. Therefore to present a true perspective of a photograph, it should ideally be viewed from the 
same distance. This distance can be calculated by multiplying the focal length of the lens by the enlargement factor.

Problems often arises when the determination of the distance at which the antemortem photograph was taken is required. If this distance and the type of camera used are unknown, establishment of those criteria becomes guess-work on the photographic format and depends on the expertise of the operator. The limits of this technique are the matching of the perspective of the two images which is of a great importance. If the condition that the image of the skull can be taken under the same conditions as the original photograph, a consistent perspective will be incorporated in each photograph for a valid comparison $(9,11)$.

The technique described by Furue relies on two important factors, firstly that a life-sized antemortem photograph of the suspected person can be obtained and secondly that the exact distance and camera specifications used to take the antemortem photograph are known. Furue was able to carry out the superimposition with full advantage of this knowledge because the details were always known and set under standard conditions in the United States Military Personnel identification photographs, which he used. Therefore this technique was able to fully reproduce the perspective exactly (2). However, this advantage is not available in all cases especially as most photographs available are domestic photographs, which are taken in a variety of unknown conditions. It may seem remarkable that successful superimposition is however possible considering the inadequacies during the superimposition technique. This reflects on the individuality of the human skull and reinforces the view that no two skulls are alike.

\section{CONCLUSION}

Craniofacial superimposition can be considered reliable provided a number of criteria are carefully observed. The photographic equipment, which provides for adequate resolution and detail for an enlarged life-sized face must be employed. Care must be taken in ensuring the proper alignment of the skull in the setup. A thorough understanding of the craniofacial anatomy, soft tissue thickness and relationships and most importantly total professional impartiality are essential elements in preventing wrongful identification.

The overall reliability of the technique can be enhanced by the consideration of several additional factors; some of which merely make the comparison easier to achieve while others impact directly on the accuracy of the results. For example, the selection of a good quality antemortem photograph will make the comparison of the images more reliable. Poor quality photographs can result in an image with poor definition because the antemortem photograph usually has to be enlarged. This can make determination of certain features difficult. It is advisable to use more than one photograph showing the face from different viewpoints, so that a series of comparisons can be made before a final decision is made. Furthermore it is preferable that the image of the face in the photograph should be as near to the centre of the photograph as possible. A photograph with the face on the outer extremities of the frame should be avoided due to barrel or pin cushion distortion as the proportions of the face will be considerably distorted. The face should be in good focus, well lit and not in a partial shadow (2).

If possible, the exact photographic conditions under which the antemortem photograph was taken should be discovered. Of particular importance are the distances at which it was taken and the focal length of the lens used to record the image. The settings used on the darkroom enlarger to produce the final print may also be of value. If these details cannot be discovered, approximations can be made. These approximate values can be used but the accuracy of the superimposition will be affected. The surface to which the antemortem photograph is attached should also be exactly parallel to the image plane of the camera. The method of mounting and orientating the skull should provide accurate and reproducible movement in small increments.

As detailed earlier, the camera must be placed at the same distance from the skull as that from which the antemortem photograph was taken. Easier and more reliable distance determination and parallelism is achieved if the cameras could be mounted on a stable fixed track rather than on tripods.

It must be borne in mind that an expert bases the reliability of craniofacial superimposition on subjective assessments. Anatomical landmarks, racial features, average tissue thickness, scar or injuries can be used as a basis for identification. However, unavoidable distortions and variances still do persist from a number of sources during the identification process, such as:

1) The photograph is not recent enough. Human morphology is never static and changes do occur in facial form over time, especially during the growth phases of puberty and as such, the quantity and quality of these changes have to be taken into account.

2) Distortions during the recording, life-size enlargement and production of the image by the operator.

3) Postmortem changes to the skull such as soil pressure, or loss of certain fragments, erosion by natural forces and chemicals.

Based on this research, employing photographic superimposition must be carefully pursued as the only means of confirming the identity of an unknown skull, unless there are unique features noticed on the skull. However it can become as an adjunct or corroborative evidence with other scientific methods of 
identification. Matching of individual facial features of a missing person with an unknown skull may be carried out by the Furue's superimposition technique. It is also particularly useful when part of the facial image is obscured or covered by a hat or mask or even helmet. This is especially so when working with images from security cameras. Further work should address the need to introduce further objectivity into the comparison of the two images using any modern technology. This will further enhance the reliability of the technique and its unchallenged acceptance in courts of law.

\section{ACKNOWLEDGEMENT}

Grateful acknowledgement is made to the Japan International Cooperation Agency (JICA) for providing the equipment for this project. We are also thankful to University of Malaya for providing a short-term research grant -Vote $F$ to buy the necessary accessories. Sincere thanks to Assistant Professor Dr. Masatsugu Hashimoto, Tokyo Dental College for his many varied assistance for this research.

\section{REFERENCE}

1) Caldwell MC. The relationship of the details of the human face to the skull and its application in forensic anthropology. Arizona State University. 1981. Thesis for the Master of Arts degree.

2) Taylor JA. Distortion in craniofacial video superimposition. University of Adelaide. 1991. Thesis for the degree of Master of Science in Dentistry.
3) Taylor JA, Brown KA. Superimposition techniques. In: Clement JG, Ranson DL eds. $1^{\text {st }}$ Edition. New York. Oxford University Press 1998; pp 151-164.

4) Glaister J \& Brash JC. Medico-legal aspects of Ruxton case. Edinburgh, E. \& S. Livingstone 1937.

5) Cocks FB. The Barklay Highway murder. Australian Police Journal 1970; 24: 173-185.

6) Thomas CJ, Nortje CJ \& van Ieperen L. A case of skull identification by photographic superimposition. J For Odontostomatol 1986; 4: 61-66.

7) Klonaris NS \& Furue T. Photographic superimposition in dental identification: Is a picture worth a thousand words? J For Sci 1980; 25: 859-865.

8) Brown KA. Developments in craniofacial superimposition for identification. J For Odontostomatol 1983; 1: 57-64.

9) Hashimoto M. A study of superimposition technique as a positive method identifacation method. Shikawa Gahuko 1992; 92: 409-34.

10) Hedgecoe J. The Photographer's handbook. London. Darling Kingsley Ltd. 1982.

11) Helmer RP. Identification of the cadaver remains of Josef Mengele. J For Sci 1987; 32: 1622-1644. 